Bull. Korean Math. Soc. 52 (2015), No. 2, pp. 421-438

http://dx.doi.org/10.4134/BKMS.2015.52.2.421

\title{
NONLINEAR DIFFERENTIAL INCLUSIONS OF SEMIMONOTONE AND CONDENSING TYPE IN HILBERT SPACES
}

\author{
Hossein Abedi and Ruhollah Jahanipur
}

\begin{abstract}
In this paper, we study the existence of classical and generalized solutions for nonlinear differential inclusions $x^{\prime}(t) \in F(t, x(t))$ in Hilbert spaces in which the multifunction $F$ on the right-hand side is hemicontinuous and satisfies the semimonotone condition or is condensing. Our existence results are obtained via the selection and fixed point methods by reducing the problem to an ordinary differential equation. We first prove the existence theorem in finite dimensional spaces and then we generalize the results to the infinite dimensional separable Hilbert spaces. Then we apply the results to prove the existence of the mild solution for semilinear evolution inclusions. At last, we give an example to illustrate the results obtained in the paper.
\end{abstract}

\section{Introduction}

Our aim is to study the nonlinear differential inclusion of first order

$$
\begin{aligned}
& x^{\prime}(t) \in F(t, x(t)), \\
& x(0)=x_{0},
\end{aligned}
$$

in a Hilbert spaces in which $F$ may be condensing or semimonotone set-valued map. An approach to investigating the existence of solution for a differential inclusion is to reduce it to a problem for ordinary differential equations. In this setting, we are interested to know under what conditions the solution of the corresponding ordinary differential equation belongs to the right side of the given differential inclusion; in other words, under what conditions a continuous function such as $f(\cdot, \cdot)$ exists so that $f(t, x) \in F(t, x)$ for every $(t, x)$ and $x^{\prime}(t)=$ $f(t, x(t))$. Thus, fixed point theorems and selection theorems to some of which we point out in Section 2, are the main tools in this method. The corresponding ordinary differential equation $x^{\prime}(t)=f(t, x(t))$ with $x(0)=x_{0}$, in case that $f: \mathbb{R} \times \mathbb{R}^{n} \rightarrow \mathbb{R}^{n}$ is continuous and Lipschitz (or Locally Lipschitz) with

Received September 28, 2013.

2010 Mathematics Subject Classification. Primary 34A60; Secondary 49K24, 47H05.

Key words and phrases. differential inclusions, set-valued integral, semimonotone and hemicontinuous multifunctions, condensing multifunctions. 
respect to the second variable, according to the Picard-Lindelof theorem [1], has a unique classical solution (local solution). However, it is well-known that continuity condition alone does not suffice for the problem to have even a local solution in infinite dimensional spaces. In a more general case, the function $f$ is considered to satisfy Caratheodory condition and therefore, solutions of Caratheodory type are obtained. To study the semilinear and nonlinear Cauchy differential equations in Hilbert spaces, we refer to [9, 10, 19, 20, 22].

For differential inclusions, the conditions we impose on set-valued map $F$ are usually a combination of two types: First, regularity of the map $F$ such as various kinds of continuity, semicontinuity and monotonicity condition. Second, geometrical conditions such as compactness, connectedness and convexity of the values of $F$. Existence of solution for differential inclusions have been studied by many authors in the past half century with different application-directed motivations, including the issues of control and optimization, dynamical systems and even biological sciences $[11,12,15,16,21]$. In the earlier works, set-valued maps have been usually considered with convex values [6, 7]. Next, in the case that the images are nonconvex, the existence theorems were also studied by some authors. For example, Bressan [7] proved the existence theorems when the values of set-valued map are completely disconnected subsets of a finite dimensional space. Also, in [2,3] differential inclusion with convex and nonconvex values on infinite dimensional Banach spaces have systematically been studied. In the case that set-valued map $F$ is nonexpansive or Lipschitz in Hausdorff metric topology, several inequalities such as the Gronwall inequality give us a lot of useful information about properties of the set of trajectories of solutions $[2,7,21,24]$. However, nonexpansive and Lipschitz conditions are very strong and are not of practical importance. For example, in optimal control problems, set-valued functions are often defined as $F(t, x)=\{f(t, x, u): u \in U\}$, where $U$ is a metric space and the single-valued function $f$ is defined on $[0, T] \times \mathbb{R}^{n} \times U$ into $\mathbb{R}^{n}$. Here $F$ need not be Lipschitz even if the function $f$ is. Thus, replacing Lipschitz condition with a weaker one, would be very valuable.

When the set-valued map $A=-F$ is maximal monotone on an infinite dimensional Hilbert space, the resolvent map $J_{\lambda}=(I+\lambda A)^{-1}$ and the Yosida approximation $A_{\lambda}=\frac{1}{\lambda}\left(I-J_{\lambda}\right)$ of $A$ are single-valued. Also, $\lim _{\lambda \rightarrow 0} A_{\lambda}(x) \in A(x)$ for every $x \in D\left(J_{\lambda}\right)$. In this case, like nonlinear differential equations with maximal monotone condition [23], first, a set of approximate solutions $x_{\lambda}$ are obtained for differential equations $x_{\lambda}^{\prime}(t)=A_{\lambda}\left(x_{\lambda}(t)\right)$. The images of these solutions under $A_{\lambda}$ constitute a weakly compact subset of the space $L^{\infty}(0, T ; H)$ and finally a Cauchy subsequence $\left\{x_{\lambda_{n}}\right\}_{n=1}^{\infty}$ is extracted such that $x_{\lambda_{n}}^{\prime}$ converges weakly to $x^{\prime}$ in $L^{2}(0, T ; H)$ and $x_{\lambda_{n}}$ converges strongly to $x$ in $L^{2}(0, T ; H)$. Since $A$ is semiclosed, this subsequence is convergent to the unique solution $x$ of (1.1).

In this paper, we establish the existence and uniqueness of the classical and generalized solution for monotone-type differential inclusion (1.1). Our 
novelty is mainly in the conditions we will impose on the nonlinearity $F$ and in the method we will use to prove the existence result which is, in fact, based upon continuous and measurable selection theorems and Kakutani's fixed point theorem.

The paper is organized as follows. In Section 2, we provide some definitions and preliminaries that are required in the next sections. We have allocated Sections 3 and 4 to the existence of generalized solution for problem (1.1). In these sections, we aim to present our main theorems and results when the multifunction $F$ is semimonotone hemicontinuous or condensing. Section 5 includes an application of the results of the previous sections to the existence of the mild solution for semilinear differential inclusions. Finally, we give an example.

\section{Definitions and preliminaries}

Let $X$ be a Banach space. We assume that $P(X)$ is the family of all non-empty subsets of $X$ and $P_{c}(X)$ (resp. $P_{c, v}(X), P_{k, v}(X)$ and $P_{b, c, v}(X)$ ) is the family of all non-empty closed (resp. closed convex, compact convex and bounded closed convex) subsets of $X$. A set-valued map $F: X \rightarrow P(Y)$ where $Y$ is another Banach space, is called upper semicontinuous (u.s.c) if for every open subset $U$ of $Y$, the set $\{x \in X: F(x) \subseteq U\}$ is open in $X$. By Proposition 1.2.5 of [18], upper semicontinuity is equivalent to the following: for each sequence $\left\{x_{n}\right\}_{n=1}^{\infty}$ in $X$ such that $x_{n} \rightarrow x$ as $n \rightarrow \infty$ and for every $\varepsilon>0$, there exists a positive integer $N$ such that

$$
F\left(x_{n}\right) \subseteq F(x)+\varepsilon B, \quad \forall n \geq N,
$$

where $B$ is the open unit ball in $Y$. The map $F$ is called lower semicontinuous (l.s.c) if for every open subset $U$ of $Y$, the set $\{x \in X: F(x) \cap U \neq \emptyset\}$ is open in $X$. By Proposition 1.2.6 of [18], lower semicontinuity is equivalent to the following: for each sequence $\left\{x_{n}\right\}_{n=1}^{\infty}$ in $X$ which converges to $x$ and for each $y \in F(x)$, there exists a sequence $\left\{y_{n}\right\}_{n=1}^{\infty}$ in $Y$ such that $y_{n} \in F\left(x_{n}\right)$ for all $n$ and $y_{n} \rightarrow y$ as $n \rightarrow \infty$. Moreover, the set-valued map $F$ is called closed when its graph $\{(x, y): y \in F(x)\}$ is closed in the product space $X \times Y$ and it is called compact if $\overline{F(X)}$ is a compact subset of $Y$. If for each $x \in X$ there exists a neighborhood $U_{x}$ of $x$ such that $F\left(U_{x}\right)$ is relatively compact in $Y$, then $F$ is called locally compact. In this paper, we apply Proposition 1.2 .23 of [18] to prove upper semicontinuity of set-valued maps:

Proposition 2.1. Let $F: X \rightarrow P_{c}(Y)$ be closed and locally compact. Then $F$ is u.s.c.

Suppose that $T>0$ and $I=[0, T]$ is an interval on the real line equipped with the $\sigma$-field of Lebesgue measurable sets. The set-valued map $F: I \rightarrow$ $P(X)$ is called measurable if for every open subset $U$ of $X$, the set $\{t \in I$ : $F(t) \cap U \neq \emptyset\}$ is measurable in $I$. To say that $F$ is measurable is the same as 
for every $x \in X$, the map $y: I \rightarrow \mathbb{R}$ defined by $y(t)=d(x, F(t))=\inf \{d(x, y)$ : $y \in F(t)\}$ is measurable [18].

A single-valued function $f: X \rightarrow Y$ is called a selection for the multifunction $F: X \rightarrow P(Y)$ provided that $f(x) \in F(x)$ for all $x \in X$. According to the Michael selection theorem [18], if $X$ is a metric and $Y$ is a Banach space, then any l.s.c set-valued map $F: X \rightarrow P_{c, v}(Y)$ has a continuous selection. Moreover, if $X$ is separable, then there exists a sequence of continuous selections $\left\{f_{n}\right\}_{n=1}^{\infty}$ such that for every $x \in X, F(x)=\overline{\left\{f_{n}(x)\right\}}$. One may also be interested in measurable selections as we do in this paper. We use the Kuratowski-Ryll Nardzewski selection theorem [18] which says that if $X$ is a separable complete metric space and the set-valued map $F: I \rightarrow P_{c}(X)$ is measurable, then $F$ has a measurable selection.

A two-variable multifunction $F: I \times X \rightarrow P(Y)$ is said to satisfy the Caratheodory condition, if (i) for every $x \in X$, the function $t \rightarrow F(t, x)$ is measurable; (ii) for a.e. $t \in I$, the function $x \mapsto F(t, x)$ is u.s.c. The next theorem which is a parametric version of Michael selection theorem, provides the conditions under which a set-valued map has a selection of Caratheodory type on a separable Banach space.

Theorem 2.2 ([18]). Let $Y$ be a separable Banach space and the set-valued map $F: I \times X \rightarrow P_{c, v}(Y)$ satisfy the following hypotheses:

i) The map $(t, x) \mapsto F(t, x)$ is measurable;

ii) For a.e. $t \in I$, the $\operatorname{map} x \rightarrow F(t, x)$ is l.s.c.

Then $F$ has a Caratheodory selection. In other words, there is a function $f: I \times X \rightarrow Y$ which is measurable in the first and continuous in the second variable and $f(t, x) \in F(t, x)$ for all $(t, x) \in I \times X$.

If $F: I \rightarrow P(X)$ is measurable, we denote by $S_{F}$ the family of all measurable selections of $F$ and for $p \geq 1$, we set $S_{F}^{p}=L^{p}(I, X) \cap S_{F}$. A set-valued map $F$ is called integrable if $S_{F}^{1} \neq \emptyset$. In that case, the Aumann integral of the set-valued map $F$ on $I$ is defined as

$$
\int_{I} F(t) d t=\left\{\int_{I} f(t) d t: f \in S_{F}^{1}\right\} .
$$

For the properties of Aumann integral of multifunctions, see e.g. [4] or Chapter 8 of $[3]$.

Next, we assume that $H$ is a real separable Hilbert space. Given a set-valued map $F: I \times H \rightarrow P(H)$, for each continuous function $x: I \rightarrow H$, we define

$$
S_{F, x}^{p}=\left\{v \in L^{p}(I ; H): v(t) \in F(t, x(t)) \text { for a.e. } t \in[0, T]\right\} .
$$

In view of the definition of the integral of set-valued maps, for any continuous function $x: I \rightarrow H$, we have

$$
\int_{I} F(t, x(t)) d t=\left\{\int_{I} v(t) d t: v \in S_{F, x}^{1}\right\}
$$


Consider the following initial value problem for differential inclusion

$$
\left\{\begin{array}{l}
x^{\prime}(t) \in F(t, x(t)), \quad t \in[0, T] \\
x(0)=x_{0}
\end{array}\right.
$$

Here, $x_{0} \in H$ and $F:[0, T] \times H \rightarrow P(H)$ is a given set-valued map. An absolutely continuous solution for the above differential inclusion is called classical solution. Along with the above differential inclusion, we consider the integral equation

$$
\left\{\begin{array}{l}
x(t)=x_{0}+\int_{0}^{t} v(s) d s, \quad t \in[0, T], \\
x(0)=x_{0}
\end{array}\right.
$$

where $v \in S_{F, x}^{1}$. If the map $v$ is continuous on $[0, T]$, then the solution $x$ of integral equation (2.2) is continuously differentiable on $[0, T]$. This is called the strong solution for problem (2.1). In general, a continuous solution $x$ for the integral equation (2.2) is called a generalized solution for the problem (2.1). In order to prove the existence of the generalized solution for problem (2.1), we impose the following hypotheses on set-valued map $F$ :

(H1) $F$ is semimonotone with constant $M>0$; that is, for every $x_{1}, x_{2} \in H$, $t \in[0, T]$ and $y_{1} \in F\left(t, x_{1}\right)$ and $y_{2} \in F\left(t, x_{2}\right)$, we have

$$
\left\langle x_{1}-x_{2}, y_{1}-y_{2}\right\rangle \leq M\left\|x_{1}-x_{2}\right\|^{2} ;
$$

(H2) There is a continuous function $h:[0, T] \times[0, \infty) \rightarrow[0, \infty)$ such that for each $t \in[0, T]$, the function $u \rightarrow h(t, u)$ is monotone increasing and $\|F(t, x)\| \leq h(t,\|x\|)$ for all $t \in[0, T]$ and $x \in H$;

(H3) The map $F$ is hemicontinuous; i.e., for each sequence $\left\{x_{n}\right\}_{n=1}^{\infty}$ in $H$ which is convergent to $x$ and each $t \in[0, T]$, if $y \in F(t, x)$, then there exists a sequence $\left\{y_{n}\right\}_{n=1}^{\infty}$ in $H$ such that for all $n \geq 1, y_{n} \in F\left(t, x_{n}\right)$ and $y_{n} \stackrel{w}{\rightarrow} y$ in $H$.

Remark 2.3. Note that if the set-valued map $F$ is l.s.c with respect to the second variable, then $F$ is hemicontinuous.

If the set-valued map $F$ is Caratheodory with closed convex values and satisfies hypothesis (H2), then any generalized solution of the differential inclusion (2.1) is classical solution. Indeed, since $x(t)-x(s) \in \int_{s}^{t} F(\tau, x(\tau)) d \tau$ for all $s, t \in[0, T]$, we have

$$
\|x(t)-x(s)\| \leq \int_{s}^{t}\|F(\tau, x(\tau))\| d \tau \leq \int_{s}^{t} h(\tau,\|x(\tau)\|) d \tau \leq K|t-s|
$$

for some constant $K>0$. Therefore, $x$ is a.e. differentiable. For every $\varepsilon>$ 0 , there is a positive number $\delta$ such that if $|t-s|<\delta$, then $F(s, x(s)) \subseteq$ $F(t, x(t))+\varepsilon B$ in which $B$ is the unit open ball in $H$. So,

$$
x(t)-x(s) \in \int_{s}^{t}(F(t, x(t))+\varepsilon B) d \tau=|t-s|(F(t, x(t))+\varepsilon B) .
$$


Therefore, letting $\varepsilon$ approach to zero, we obtain $x^{\prime}(t) \in F(t, x(t))$. Thus $x$ is a classical solution.

The Hausdorff measure of noncompactness on the Hilbert space $H$ is a function $\alpha: P_{b}(H) \rightarrow[0, \infty)$ which is defined by

$$
\alpha(A)=\inf \left\{\varepsilon>0: A \subseteq \bigcup_{i=1}^{n} X_{i}, \quad \operatorname{diam}\left(X_{i}\right) \leq \varepsilon\right\}, \quad \forall A \in P_{b}(H) .
$$

It is not difficult to see that for all $A, B \in P_{b}(H)$, (1) if $A \subseteq B$, then $\alpha(A) \leq$ $\alpha(B) ;(2) \alpha(A \cup B)=\max \{\alpha(A), \alpha(B)\} ;(3) \alpha(A+B) \leq \alpha(A)+\alpha(B) ;(4)$ for every $\lambda \in \mathbb{R}, \alpha(\lambda A)=|\lambda| \alpha(A)$; (5) $\alpha(\operatorname{co}(A))=\alpha(A), \alpha(\bar{A})=\alpha(A)$, where $\operatorname{co}(A)$ is the convex hull of $A$; and finally $(6) \alpha(A)=0$ if and only if $A$ is relatively compact. In general, any function on $P_{b}(H)$ with the properties $(1),(2),(3),(5)$ and (6), is called a regular measure of noncompactness on $H$. Let $Y$ be a Banach space and $\beta$ a regular measure of noncompactness on $Y$. A set-valued map $G: Y \rightarrow P(Y)$ is called $\beta$-(countable) condensing if for every (countable) bounded subset $A$ of $Y$ such that $\beta(A)>0$, we have $\beta(G(A))<\beta(A)$. In Section 3, we will need a generalized form of the Monch fixed point theorem (Theorem 4.16 of [1]) for condensing set-valued maps:

Theorem 2.4. Suppose that $C$ is a closed convex subset of the Banach space $Y$ and let the closed set-valued map $G: C \rightarrow P_{k, v}(C)$ be condensing with respect to the regular measure of noncompactness $\beta$. Then $G$ has a fixed point.

To define the measure of noncompactness for the bounded subsets of the space $C(I ; H)$ of all continuous functions $x: I \rightarrow H$ equipped with the supnorm, we set $\alpha_{C}(\Omega)=\sup _{t \in I} e^{-L t} \alpha(\Omega(t))$, for each $\Omega \in P_{b}(C(I ; H))$ where $L$ is a suitable constant to be determined latter in the paper and $\Omega(t)=\{x(t)$ : $x \in \Omega\}$. One can easily check that $\alpha_{C}$ has all properties of the measure of noncompactness except for the regularity property. In other words, if $\Omega \subseteq$ $C(I ; H)$ is a relatively compact set, then $\alpha_{C}(\Omega)=0$, but the reverse may not be hold. However, if $\Omega$ is a family of equicontinuous functions and $\alpha_{C}(\Omega)=0$, then $\Omega$ is relatively compact subset of $C(I ; H)$. To measure the equicontinuity of the subsets of $C(I ; H)$, we use the modulus of equicontinuity. This is defined as

$$
\bmod _{C}(\Omega)=\lim _{\delta \rightarrow 0} \sup _{x \in \Omega} \sup _{\left|t_{1}-t_{2}\right| \leq \delta}\left\|x\left(t_{1}\right)-x\left(t_{2}\right)\right\| .
$$

Note that $\bmod { }_{C}(\Omega)=0$ if and only if $\Omega \subseteq C(I ; H)$ is an equicontinuous family. Now, we are ready to define a regular measure of noncompactness on $C(I ; H)$ :

$$
\chi(\Omega)=\max _{D \in \Lambda(\Omega)}\left\{\alpha_{C}(D), \bmod _{C}(D)\right\}
$$

for all $\Omega \in P_{b}(C(I ; H))$. Here $\Lambda(\Omega)$ is the family of all countable subsets of $\Omega$. 


\section{Semimonotone set-valued maps}

In this section, we first prove the existence theorem for differential inclusion (1.1) when $F$ is a semimonotone set-valued map on a finite dimensional space and then we generalize it to the infinite dimensional Hilbert spaces. Note that without loss of generality, we can assume that $x(0)=0$.

Theorem 3.1. Let the Caratheodory set-valued map $F:[0, T] \times \mathbb{R}^{n} \rightarrow P_{c, v}\left(\mathbb{R}^{n}\right)$ satisfy hypothesis $(\mathrm{H} 2)$ and the differential equation $u^{\prime}=h(t, u)$ with $u(0)=0$ has a solution $u(t)$ existing on $[0, T]$. Then, the differential inclusion

$$
\left\{\begin{array}{l}
x^{\prime}(t) \in F(t, x(t)), \quad t \in[0, T] \\
x(0)=0
\end{array}\right.
$$

has a classical solution.

Proof. We are to show that there exists a function $x \in C\left(I ; \mathbb{R}^{n}\right)$ such that $x(t) \in \int_{0}^{t} F(s, x(s)) d s$ for all $t \in[0, T]$. To this end, we define the set-valued $\operatorname{map} \Lambda$ on $C\left(I ; \mathbb{R}^{n}\right)$ as

$$
\Lambda(x)=\left\{y: I \rightarrow \mathbb{R}^{n} \mid y(t)=\int_{0}^{t} v(s) d s, \forall t \in[0, T] \text { for some } v \in S_{F, x}^{1}\right\} .
$$

Since each Caratheodory map is measurable, by Kuratowski-Ryll Nardzewski selection theorem and hypothesis (H2), the map $\Lambda$ has non-null values. We consider the closed convex subset

$$
K=\left\{x \in C\left(I ; \mathbb{R}^{n}\right): x(0)=0,\|x(t)\| \leq u(t) \quad \forall t \in[0, T]\right\},
$$

of the space $C\left(I, \mathbb{R}^{n}\right)$. First, we show that $\Lambda: K \rightarrow P(K)$. Indeed, for all $t \in[0, T], x \in K$ and $v \in S_{F, x}^{1}$ we have

$$
\left\|\int_{0}^{t} v(s)\right\| \leq \int_{0}^{t}\|v(s)\| d s \leq \int_{0}^{t} h(s,\|x(s)\|) d s \leq \int_{0}^{t} h(s, u(s)) d s=u(t) .
$$

Next, it is easy to see that the values of $\Lambda$ are convex. Let $\left\{y_{n}\right\}_{n=1}^{\infty}$ be a sequence in $\Lambda(x)$ which is convergent to $y$ in $C\left(I, \mathbb{R}^{n}\right)$. Then, there exists a sequence $\left\{v_{n}\right\}_{n=1}^{\infty}$ in $S_{F, x}^{1}$ such that $y_{n}(t)=\int_{0}^{t} v_{n}(s) d s$ for all $t \in[0, T]$. Since $t \mapsto h(t, u(t))$ is a continuous function and

$$
\left\|v_{n}(t)\right\| \leq\|F(t, x(t))\| \leq h(t, u(t))
$$

for each $t \in[0, T]$ and every $n \geq 1$, we observe that $\left\{v_{n}\right\}_{n=1}^{\infty}$ is in fact a sequence of elements of the Hilbert space $L^{2}\left([0, T] ; \mathbb{R}^{n}\right)$. So, it has a subsequence (we denote it by the same symbol) that is weakly convergent to a function $v \in L^{2}\left([0, T] ; \mathbb{R}^{n}\right)$, say. Then, we conclude from Corollary V.3.14 of [14] that there is a sequence $\left\{w_{n}\right\}_{n=1}^{\infty}$ of convex combinations of $\left\{v_{1}, v_{2}, \ldots\right\}$ which is, in particular, convergent to $v$ in the norm topology of the space $L^{1}\left([0, T] ; \mathbb{R}^{n}\right)$. Since $F$ has convex values, $w_{n} \in S_{F, x}^{1}$. The sequence $\left\{w_{n}\right\}_{n=1}^{\infty}$ has a subsequence (we denote it again by the same symbol) which converges to $v$ a.e. on 
$[0, T]$. Because the values of $F$ are closed, we have $v \in S_{F, x}^{1}$ and the dominated convergence theorem gives

$$
y(t)=\lim _{n \rightarrow \infty} y_{n}(t)=\lim _{n \rightarrow \infty} \int_{0}^{t} v_{n}(s) d s=\int_{0}^{t} v(s) d s .
$$

Thus, $\Lambda(x)$ is a closed set. Now, we prove that $\Lambda$ is a closed map. Let sequences $\left\{x_{n}\right\}_{n=1}^{\infty}$ and $\left\{y_{n}\right\}_{n=1}^{\infty}$ in $K$ converge to the continuous functions $x$ and $y$ in the space $C\left(I ; \mathbb{R}^{n}\right)$, respectively and furthermore $y_{n} \in \Lambda\left(x_{n}\right)$ for all $n$. By upper semicontinuity of $F$ with respect to the second variable, for given $\varepsilon>0$, large enough $n$ and any $t \in[0, T]$, we have $F\left(t, x_{n}(t)\right) \subseteq F(t, x(t))+\varepsilon B$. So,

$$
y_{n}(t) \in \int_{0}^{t} F\left(s, x_{n}(s)\right) d s \subseteq \int_{0}^{t} F(s, x(s)) d s+t \varepsilon B .
$$

Since $\varepsilon$ was arbitrary, we obtain $y_{n}(t) \in \int_{0}^{t} F(s, x(s)) d s=\Lambda(x)$. This together with the result that $\Lambda(x)$ is closed, implies that $y(t) \in \Lambda(x)$ for all $t \in[0, T]$. Finally, we show that $\Lambda(K)$ is uniformly bounded and equicontinuous in the Banach space $C\left(I ; \mathbb{R}^{n}\right)$. Uniform boundedness of $\Lambda$ is a consequence of continuity of the function $u$ on $[0, T]$ and the following dominance relation which by hypothesis $\mathrm{H} 2$, is true for all $x \in K$ and $y \in \Lambda(x)$ :

$$
\sup _{t \in I}\|y(t)\| \leq \int_{0}^{T}\|F(s, x(s))\| d s \leq \int_{0}^{T} h(s, u(s)) d s=u(T) .
$$

Also, given $y \in \Lambda(K)$, we can prove similarly that

$$
\left\|y\left(t_{1}\right)-y\left(t_{2}\right)\right\| \leq\left|u\left(t_{1}\right)-u\left(t_{2}\right)\right|
$$

for all $t_{1}, t_{2} \in[0, T]$. Thus, by the Arzela-Ascoli theorem, $\Lambda(K)$ is a relatively compact subset of the space $C\left(I ; \mathbb{R}^{n}\right)$. So, $\Lambda$ is compact and it is also u.s.c by Proposition 2.1. Now, by Kakutani's fixed point theorem [17], $\Lambda$ has a fixed point in $K$ which is a classical solution of the differential inclusion (3.1).

In the above theorem, if we replaced the upper semicontinuity condition on $F$ by lower semicontinuity, then according to Theorem 2.2 , there is a singlevalued Caratheodory function $f:[0, T] \times \mathbb{R}^{n} \rightarrow \mathbb{R}^{n}$ such that $f(t, x) \in F(t, x)$. Then, Theorem 3.2 of [20], indicates that the ordinary differential equation

$$
\left\{\begin{array}{l}
x^{\prime}(t)=f(t, x(t)), \quad t \in[0, T] \\
x(0)=0,
\end{array}\right.
$$

has a generalized solution on $[0, T]$. Thus, the above differential inclusion has a generalized solution on $[0, T]$. So we have the following theorem.

Theorem 3.2. Let the integrable set-valued map $F:[0, T] \times \mathbb{R}^{n} \rightarrow P_{c, v}\left(\mathbb{R}^{n}\right)$ be l.s.c respect to the second variable and satisfy condition $(\mathrm{H} 2)$. If the differential equation $u^{\prime}=h(t, u)$ with $u(0)=0$ has a solution $u(t)$ existing on $[0, T]$, then the differential inclusion (3.1) has a generalized solution. 
Now, we generalize the previous theorem to infinite dimensional real separable Hilbert spaces. Denote by $C_{l_{0}}(I ; H)$ the space of all continuous functions $x: I \rightarrow H$ such that $x(0)=0$ equipped with sup-norm. Also, $L_{l_{0}}^{2}(I ; H, M)$ stands for the Hilbert space of all measurable functions $x: I \rightarrow H$ such that

$$
\int_{0}^{T} e^{-2 M t}\|x(t)\|^{2} d t<\infty
$$

with the inner product

$$
\langle x, y\rangle_{M}=\int_{0}^{T} e^{-2 M t}\langle x(t), y(t)\rangle d t,
$$

and the norm

$$
\|x\|_{M}=\left(\int_{0}^{T} e^{-2 M t}\|x(t)\|^{2} d t\right)^{\frac{1}{2}} .
$$

Proposition 3.3. Let the Caratheodory multifunction $F:[0, T] \times H \rightarrow P(H)$ satisfy hypothesis (H2). Define the set-valued map $G$ on $C_{l_{0}}(I ; H)$ as

$$
G x=\left\{y: I \rightarrow H \mid y \in S_{F, x}^{1}, y(t) \in F(t, x(t)) \text { for all } t \in I\right\} .
$$

Then

(a) $G: C_{l_{0}}(I ; H) \rightarrow P\left(L_{l_{0}}^{2}(I ; H, M)\right)$ is bounded on bounded subsets of $C_{l_{0}}(I ; H)$.

(b) If $F$ satisfies hypothesis (H1), then $G$ is semimonotone with constant $M$.

(c) If $F$ satisfies hypothesis (H3), then $G$ is hemicontinuous.

Proof. We refer to [20] for the proof of parts (a) and (b). In particular, if $B \subseteq C_{l_{0}}(I ; H)$ is a bounded set, then there exists a constant $K>0$ such that $\|G x\|_{M} \leq \gamma(T) K$, where $\gamma(T)=\frac{1}{\sqrt{2 M}}\left(1-e^{-2 M T}\right)^{\frac{1}{2}}$. To prove (c), suppose that $\left\{x_{n}\right\}_{n=1}^{\infty}$ is a sequence which converges to $x$ in $C_{l_{0}}(I ; H)$. Since $F$ is hemicontinuous, if $y \in G(x)$, then there is a sequence of functions $\left\{y_{n}\right\}_{n=1}^{\infty}$ such that $y_{n}(t) \in F\left(t, x_{n}(t)\right)$ for each $n \geq 1$ and $t \in[0, T]$ and also, $y_{n}(t) \stackrel{w}{\rightarrow} y(t)$ in $H$ as $n \rightarrow \infty$. According to hypothesis (H2), we have $\left\{y_{n}\right\}_{n=1}^{\infty} \subseteq L_{l_{0}}^{2}(I ; H, M)$ and $\left\langle y_{n}(t)-y(t), z(t)\right\rangle \rightarrow 0$ as $n \rightarrow \infty$ for every $z \in L_{l_{0}}^{2}(I ; H, M)$. Moreover, the Cauchy-Schwarz inequality implies that $\left|\left\langle y_{n}(t)-y(t), z(t)\right\rangle\right| \leq K\|z(t)\|$ for some constant $K>0$. Now, by the dominated convergence theorem, we have

$$
\left|\left\langle y_{n}-y, z\right\rangle_{M}\right| \leq \int_{0}^{T} e^{-2 M t}\left|\left\langle y_{n}(t)-y(t), z(t)\right\rangle\right| d t \rightarrow 0,
$$

as $n \rightarrow \infty$. Therefore, $y_{n} \stackrel{w}{\rightarrow} y$ in $L_{l_{0}}^{2}(I ; H, M)$ and the proof is complete.

Next, we generalize Lemma 1.2 of [9] for the set-valued hemicontinuous maps. It is needed to prove the main theorem of this section. 
Lemma 3.4. Suppose that $D$ is dense subspace of Hilbert space $H$ and the set-valued map $F: D \subseteq H \rightarrow P(H)$ satisfy hypothesis (H3). If $u_{0} \in D, y_{0} \in H$ and $M>0$ is a constant such that

$$
\left\langle y_{0}-y, u_{0}-u\right\rangle \leq M\left\|u_{0}-u\right\|^{2} \quad \text { for all } u \in D \text { and } y \in F(u),
$$

then $y_{0} \in F\left(u_{0}\right)$.

Proof. Assume that $y_{0} \notin F\left(u_{0}\right)$. Fix $y \in F\left(u_{0}\right)$. Since $y-y_{0} \neq 0$ and $D$ is dense in $H$, we can find $v_{y} \in D$ such that $\left\langle y-y_{0}, v_{y}\right\rangle>0$. Setting $u_{n}=u_{0}+\frac{1}{n} v_{y}$ for each $n \geq 1$, we have $u_{n} \in D$ and by hypothesis H3, we can take $y_{n} \in F\left(u_{n}\right)$ such that $y_{n} \stackrel{w}{\rightarrow} y$ in $H$. By $(3.2)$, we have for all $n \in \mathbb{N}$ that

Therefore,

$$
\frac{1}{n}\left\langle y_{0}-y_{n}, v_{y}\right\rangle \geq-M \frac{\left\|v_{y}\right\|^{2}}{n^{2}} \text {. }
$$

$$
\left\langle y-y_{n}, v_{y}\right\rangle \geq\left\langle y-y_{0}, v_{y}\right\rangle-M \frac{\left\|v_{y}\right\|^{2}}{n} .
$$

Now, letting $n \rightarrow \infty$, we obtain $0<\left\langle y-y_{0}, v_{y}\right\rangle \leq 0$. This is a contradiction.

Our main theorem in this section extends Theorem 3.1 and 3.2 to differential inclusions on infinite dimensional real separable Hilbert spaces.

Theorem 3.5. Let $H$ be a real separable Hilbert space and the Caratheodory set-valued map $F:[0, T] \times H \rightarrow P_{c, v}(H)$ satisfy hypotheses $(\mathrm{H} 1)-(\mathrm{H} 3)$. If the differential equation $u^{\prime}=h(t, u)$ with $u(0)=0$ has a solution $u(t)$ existing on $[0, T]$, then the differential inclusion problem

$$
\left\{\begin{array}{l}
x^{\prime}(t) \in F(t, x(t)), \quad t \in[0, T], \\
x(0)=0,
\end{array}\right.
$$

has a unique classical solution.

Proof. Take an orthonormal basis $\left\{e_{n}\right\}_{n=1}^{\infty}$ for $H$ and let $H_{n}$ be the subspace of $H$ generated by $\left\{e_{1}, \ldots, e_{n}\right\}$. Then, $\left\{H_{n}\right\}_{n=1}^{\infty}$ is an increasing sequence of finite dimensional subspaces of $H$ with the property $\overline{\cup_{n=1}^{\infty} H_{n}}=H$. If $P_{n}$ is the orthogonal projection of $H$ onto $H_{n}$, then each $P_{n}$ induces a corresponding projection of $L_{l_{0}}^{2}(I ; H, M)$ onto subspace $L_{l_{0}}^{2}\left(I ; H_{n}, M\right)$, we denote it again by $P_{n}$ :

$$
\left(P_{n} u\right)(t)=P_{n} u(t) \in H_{n}, \quad \forall t \in I, u \in L_{l_{0}}^{2}(I ; H, M) .
$$

We have $P_{n}^{*}=P_{n} \rightarrow I$ as $n \rightarrow \infty$ where $P_{n}^{*}$ is the adjoint operator of $P$ and $I$ is the identity map on $H$. For each $n \geq 1$, we define the set-valued map $P_{n} F:[0, T] \times H_{n} \rightarrow P_{c, v}\left(H_{n}\right)$ by $P_{n} F(t, x)=\left\{P_{n}(y) \mid y \in F(t, x)\right\}$. Theorem 1.2 .8 of [21] yields that $P_{n} F$ is a Caratheodory map and since

$$
\left\|P_{n} F(t, x)\right\|=\sup _{y \in F(t, x)}\left\|P_{n} y\right\| \leq\|F(t, x)\| \leq h(t,\|x\|),
$$


it also satisfies hypothesis (H2). By Theorem 3.1, for each $n \geq 1$, the differential inclusion

$$
\left\{\begin{array}{l}
x^{\prime}(t) \in P_{n} F(t, x(t)), \quad t \in[0, T] \\
x(0)=0
\end{array}\right.
$$

has a classical solution $x_{n}:[0, T] \rightarrow H_{n}$. On the other hand, hypothesis (H1) holds for $P_{n} F$. In fact, let $x_{i} \in H_{n}$ and $y_{i} \in P_{n} F\left(t, x_{i}\right)$ for $i=1,2$. Then there exist $z_{i} \in F\left(t, x_{i}\right), i=1,2$, such that $y_{i}=P_{n} z_{i}$. Since $P_{n}=P_{n}^{*}$, we get

$$
\left\langle y_{2}-y_{1}, x_{2}-x_{1}\right\rangle=\left\langle z_{2}-z_{1}, x_{2}-x_{1}\right\rangle \leq M\left\|x_{2}-x_{1}\right\|^{2} .
$$

Thus, just as the proof of Proposition 3.5 of [20], the sequence $\left\{x_{n}\right\}_{n=1}^{\infty}$ is bounded by a constant which depends only on $F$ and $T$ and we have

$$
\left\|x_{n}\right\|_{\infty}^{2} \leq e^{(2 M+1) T} \int_{0}^{T}\|F(s, 0)\|^{2} d s .
$$

Therefore, according to part (a) of Proposition 3.3, $G\left(\left\{x_{n}\right\}_{1}^{\infty}\right)$ is a bounded set in $L_{l_{0}}^{2}(I ; H, M)$. Now, we define the linear operator $\Gamma: L_{l_{0}}^{2}(I ; H, M) \rightarrow$ $C_{l_{0}}(I ; H)$ by

Since

$$
(\Gamma y)(t)=\int_{0}^{t} y(s) d s
$$

$$
\|(\Gamma y)(t)\| \leq \gamma(T)\left(\int_{0}^{T} e^{-2 M t}\|y(t)\|^{2} d t\right)^{\frac{1}{2}}=\gamma(T)\|y\|_{M},
$$

where $\gamma(T)=\frac{1}{\sqrt{2 M}}\left(1-e^{-2 M T}\right)^{\frac{1}{2}}, \Gamma$ is continuous. Consider the set-valued map $\Gamma P_{n} G x=\left\{\Gamma y \mid y \in P_{n} G x\right\}$ from $C_{l_{0}}(I ; H)$ into $C_{l_{0}}\left(I ; H_{n}\right)$. Let $x_{n}$ be a solution of the differential inclusion (3.4), so that $x_{n} \in \Gamma P_{n} G x_{n}$ for all $n \geq 1$. We can find $y_{n} \in L_{l_{0}}^{2}(I ; H, M)$ such that $x_{n}=\Gamma P_{n} y_{n}$. Since $y_{n} \in G x_{n}$, the sequence $\left\{y_{n}\right\}_{n=1}^{\infty}$ is bounded in $L_{l_{0}}^{2}(I ; H, M)$ and it is possible to extract a subsequence (we denote it again by $\left\{y_{n}\right\}_{n=1}^{\infty}$ ) which is weakly convergent in $L_{l_{0}}^{2}(I ; H, M)$. Suppose that $y_{n} \stackrel{w}{\rightarrow} y$ as $n \rightarrow \infty$. Then, $x_{n} \stackrel{w}{\rightarrow} \Gamma y=x \in$ $C_{l_{0}}(I ; H)$. It is enough to show that $y \in G x$. With the same method as in the proof of Theorem 3.6 of [20], one can show that

$$
\langle w-y, x-u\rangle_{M} \geq-M\|x-u\|_{M}^{2}, \quad \forall u \in C_{l_{0}}(I ;, H), w \in G u .
$$

Since, by Proposition 3.3, $G$ is hemicontinuous, the result follows from the Lemma 3.4. Finally, we come to prove the uniqueness of solution. If $x^{1}$ and $x^{2}$ are two solutions of problem (3.3), then we have $x^{2}(t)-x^{1}(t)=$ $\int_{0}^{t}\left(v_{1}(s)-v_{2}(s)\right) d s$ for some $v_{1} \in S_{F, x^{1}}^{1}$ and $v_{2} \in S_{F, x^{2}}^{1}$ and Lemma 3.4 of [20] shows that

$$
\left\|x^{2}(t)-x^{1}(t)\right\|^{2} \leq 2 M \int_{0}^{t}\left\|x^{2}(s)-x^{1}(s)\right\|^{2} d s .
$$

Thus, by the Gronwall inequality, $\left\|x^{2}(t)-x^{1}(t)\right\|=0$ for all $t \in[0, T]$. 
Now, if $F$ is integrable hemicontinuous in the previous theorem, then one can easy see that the set-valued map $P_{n} F:[0, T] \times H \rightarrow P_{c, v}\left(H_{n}\right)$ is hemicontinuous. So, $P_{n} F$ is l.s.c with respect to the second variable, since weak and strong convergence are equivalent on $H_{n}$. Then, by Theorem 3.2 the differential inclusion (3.4) has a generalized solution $x_{n}$ for all $n$. Therefore, with a similar proof as Theorem 3.5 we obtain the following theorem.

Theorem 3.6. Let $H$ be a real separable Hilbert space and the integrable setvalued map $F:[0, T] \times H \rightarrow P_{c, v}(H)$ satisfy hypotheses $(\mathrm{H} 1)-(\mathrm{H} 3)$. If the differential equation $u^{\prime}=h(t, u)$ with $u(0)=0$ has a solution $u(t)$ existing on $[0, T]$, then the differential inclusion (3.3) has a unique generalized solution.

\section{Condensing set-valued maps}

In this section, we discuss the existence of a solution for differential inclusion (3.3) in the case that the set-valued map $F$ is condensing with respect to a regular measure of noncompactness on a real separable Hilbert space. we get a global existence theorem that is the analogous results of the Theorems 5.2.1 of [21], under a continuous bounded. Our main result in this section is the following

Theorem 4.1. Let $H$ be a real separable Hilbert space. Suppose that the Caratheodory (integrable) set-valued map $F:[0, T] \times H \rightarrow P_{c, v}(H)$ satisfy hypotheses (H2) and there is a function $k \in L^{1}([0, T] ;[0, \infty))$ such that for every countable pointwise bounded subset $\Omega$ of $C(I ; H)$, we have

$$
\alpha(F(t, \Omega(t))) \leq k(t) \alpha(\Omega(t)),
$$

where $\alpha$ is the Hausdorff measure of noncompactness. If the differential equation $u^{\prime}=h(t, u)$ with $u(0)=0$ has a solution $u(t)$ existing on $[0, T]$, then the differential inclusion (3.3) has a classical (generalized) solution on $[0, T]$.

To prove the theorem, we need a very useful lemma the proof of which can be found in [21]. consider the set $K \subset C(I, H)$ as

$$
K=\{x \in C(I ; H): \quad x(0)=0, \quad\|x(t)\| \leq u(t) \quad \forall t \in[0, T]\} .
$$

Lemma 4.2. Suppose that all conditions of Theorem 4.1 hold. Let $\left\{x_{n}\right\}_{n=1}^{\infty}$ be a sequence in $K$ which converges to $x$ and $\left\{v_{n}\right\}_{n=1}^{\infty}$ be a sequence in $L^{1}([0, T] ; H)$ such that $v_{n} \stackrel{w}{\rightarrow} v$ in $L^{1}([0, T] ; H)$. If for each $n \geq 1$ and for a.e. $t \in[0, T]$, $v_{n}(t) \in F\left(t, x_{n}(t)\right)$, then $v \in S_{F, x}^{1}$. Moreover, $\int_{0}^{t} v_{n}(s) d s$ tends to $\int_{0}^{t} v(s) d s$ in the space $C(I ; H)$

Proof. Consider the $K \subseteq C(I ; H)$ as above and the set-valued map $\Lambda$ introduced in the proof of Theorem 3.1. Since $H$ is separable, $\Lambda$ has nonnull values and according to the hypotheses $(\mathrm{H} 2),\|\Lambda(x)(t)\| \leq u(t)$ for all $(t, x) \in[0, T] \times K$. First, we show that $\Lambda$ is closed. To this end, let the sequences $\left\{x_{n}\right\}_{n=1}^{\infty}$ and $\left\{y_{n}\right\}_{n=1}^{\infty}$ converge to $x$ and $y$, respectively, in the space 
$C(I ; H)$ and $y_{n} \in \Lambda\left(x_{n}\right)$ for all $n \in \mathbb{N}$. So, there exists a sequence of functions $\left\{v_{n}\right\}_{n=1}^{\infty}$ in $L^{1}([0, T] ; H)$ such that $v_{n} \in S_{F, x_{n}}^{1}$ for each $n \in \mathbb{N}$ and

$$
y_{n}(t)=\int_{0}^{t} v_{n}(s) d s, \quad \forall t \in[0, T] .
$$

We obtain from hypothesis (H2) that $\left\|v_{n}(t)\right\| \leq\left\|F\left(t, x_{n}(t)\right)\right\| \leq h\left(t,\left\|x_{n}(t)\right\|\right) \leq$ $h(t, u(t))$. Thus, $\left\{v_{n}\right\}_{n=1}^{\infty}$ is integrably bounded and moreover since $h$ is continuous, $\left\{v_{n}(t)\right\}_{n=1}^{\infty}$ is relatively weakly compact in $H$ for all $t \in[0, T]$. Therefore, we can assume that $v_{n} \stackrel{w}{\rightarrow} v$ in $L^{1}([0, T] ; H$ ) (see [5]). Now, by Lemma 4.2, $v \in S_{F, x}^{1}$ and $y_{n}(t) \rightarrow \int_{0}^{t} v(s) d s$ in $C([0, T] ; H)$ as $n \rightarrow \infty$. Consequently, we have $y(t)=\int_{0}^{t} v(s) d s$. This proves that $\Lambda$ is a closed set-valued map and a similar argument indicates that $\Lambda(x)$ is also closed for every $x \in K$. Next, we show that $\Lambda(x)$ is compact for all $x \in K$. Given any sequence $\left\{y_{n}\right\}_{n=1}^{\infty} \subseteq \Lambda(x)$, we can find for every $n \geq 1$, a $v_{n} \in S_{F, x}^{1}$ such that $y_{n}(t)=\int_{0}^{t} v_{n}(s) d s$ for all $t \in[0, T]$. Again passing to a suitable subsequence, if necessary, we may assume that $v_{n} \stackrel{w}{\rightarrow} v$ in $L^{1}(I ; H)$. Thus, by Lemma $4.2, \int_{0}^{t} v_{n}(s) d s \stackrel{n \rightarrow \infty}{\rightarrow} \int_{0}^{t} v(s) d s$ in $C([0, T] ; H)$. Therefore, $\Lambda(x)$ is compact. Now, let us assume that $\chi$ is a regular measure of noncompactness we defined at the end of Section 2. Assume that $\Omega \subseteq C(I ; H)$ is bounded and $\chi(\Omega) \leq \chi(\Lambda(\Omega))$. If $\left\{x_{n}\right\}_{n=1}^{\infty}$ is a sequence in $\Omega$, then $\sup _{t \in I} e^{-L t} \alpha\left(\left\{x_{n}(t)\right\}_{n=1}^{\infty}\right) \leq \sup _{t \in I} e^{-L t} \alpha\left(\left\{y_{n}(t)\right\}_{n=1}^{\infty}\right)$, where $y_{n} \in \Lambda\left(x_{n}\right)$. Therefore, we obtain

$$
\begin{aligned}
& \sup _{t \in[0, T]}\left(e^{-L t} \alpha\left(\left\{x_{n}(t)\right\}_{n=1}^{\infty}\right)\right) \\
\leq & \sup _{t \in[0, T]}\left(e^{-L t} \alpha\left(\left\{\int_{0}^{t} v_{n}(s) d s\right\}_{n=1}^{\infty}\right)\right) \\
\leq & \sup _{t \in[0, T]}\left(e^{-L t} \sup _{u \in[0, T]}\left(e^{-L u} \alpha\left(\left\{x_{n}(u)\right\}_{n=1}^{\infty}\right) \int_{0}^{t} e^{L s} k(s) d s\right)\right) \\
\leq & \sup _{u \in[0, T]}\left(e^{-L u} \alpha\left(\left\{x_{n}(u)\right\}_{n=1}^{\infty}\right)\right) \sup _{t \in[0, T]}\left(\int_{0}^{t} e^{-L(t-s)} k(s) d s\right) .
\end{aligned}
$$

If we choose constant $L$ in such a way that $\sup _{t \in[0, T]} \int_{0}^{t} e^{-L(t-s)} k(s) d s<$ 1 , then we obtain $\alpha_{C}\left(\left\{x_{n}\right\}_{n=1}^{\infty}\right)=0$. Note that since $\left\{y_{n}\right\}_{n=1}^{\infty}$ is a relatively compact subset of $C([0, T] ; H)$, then $\alpha_{C}\left(\left\{y_{n}\right\}_{n=1}^{\infty}\right)=0$. Moreover, $\bmod _{C}\left(\left\{y_{n}\right\}_{n=1}^{\infty}\right)=0$. Thus, $\chi(\Lambda(\Omega))=0$ and consequently, $\chi(\Omega)=0$. This shows that the set-valued map $\Lambda$ is $\chi$ - condensing. Therefore, according to Theorem $2.4, \Lambda$ has a fixed point which is a generalized solution of the differential inclusion (3.3). 


\section{Mild solutions}

In this section, we come to prove the existence of mild solution for semilinear differential inclusion

$$
\left\{\begin{array}{l}
x^{\prime}(t)-A x(t) \in F(t, x(t)), \quad t \in[0, T] \\
x(0)=\xi
\end{array}\right.
$$

with $\xi \in H$. By a mild solution to (5.1), we mean a continuous function $x:[0, T] \rightarrow H$ such that for every $t \in[0, T]$,

$$
x(t)=S(t) \xi+\int_{0}^{t} S(t-s) v(s) d s,
$$

where $v \in S_{F, x}^{1}$. If for a.e. $t \in[0, T], v(t) \in D(A)$ and the function $t \mapsto A v(t)$ is integrable, then for every $\xi \in D(A)$, the mild solution is absolutely continuous and therefore, almost- everywhere differentiable.

Theorem 5.1. Suppose that $H$ is a separable Hilbert space and $F:[0, T] \times H \rightarrow$ $P_{c, v}(H)$ is a integrable (Caratheodory) set-valued map satisfying hypotheses (H1)-(H3). If $A: D(A) \subseteq H \rightarrow H$ is the infinitesimal generator of a contraction $C_{0}$ semigroup $\{S(t)\}_{t>0}$ and the differential equation $u^{\prime}=h(t, u)$ with $u(0)=0$ has a solution $u(t)$ existing on $[0, T]$, then the semilinear differential inclusion (5.1) has a unique mild solution.

Proof. By the Hille-Yosida theorem, we have for every $n \in \mathbb{N}$ that

$$
\left\|(n I-A)^{-1}\right\| \leq \frac{1}{n} .
$$

Define $R_{n}=n(n I-A)^{-1}$ and $A_{n}=A R_{n}$. Since $A_{n} x=\left(n R_{n}-n I\right) x$ for each $x \in H$, we have $\left\|A_{n}\right\| \leq 2 n$. Therefore, the bounded linear operator $A_{n}$ is the infinitesimal generator of the uniformly continuous semigroup $e^{A_{n} t}$. Then, we define the set-valued map $F_{n}:[0, T] \times H \rightarrow P_{c, v}(H)$ by $F_{n}(t, x)=A_{n} x+F(t, x)$. We show that $F_{n}$ satisfies hypotheses (H1)-(H3). Since by the Lumer-Philips theorem, $A$ is dissipative and $R_{n} x \in D(A)$ for every $x \in H$, we have

$$
\begin{aligned}
\left\langle A_{n} x, x\right\rangle=\left\langle A R_{n} x, x\right\rangle & =\left\langle A R_{n} x, R_{n} x\right\rangle-\frac{1}{n}\left\langle A R_{n} x, A R_{n} x\right\rangle \\
& \leq-\frac{1}{n}\left\|A R_{n} x\right\|^{2} \leq 0 .
\end{aligned}
$$

Therefore, $A_{n}$ is also dissipative. Take $x_{1}, x_{2} \in H, t \in[0, T]$ and $y_{1} \in F_{n}\left(t, x_{1}\right)$, $y_{2} \in F_{n}\left(t, x_{2}\right)$. Then, there are $z_{1} \in F\left(t, x_{1}\right)$ and $z_{2} \in F\left(t, x_{2}\right)$ such that $y_{i}=A_{n} x_{i}+z_{i}$ for $i=1,2$. So, we obtain

$$
\begin{aligned}
\left\langle y_{1}-y_{2}, x_{1}-x_{2}\right\rangle & =\left\langle A_{n} x_{1}+z_{1}-A_{n} x_{2}-z_{2}, x_{1}-x_{2}\right\rangle \\
& =\left\langle A_{n}\left(x_{1}-x_{2}\right), x_{1}-x_{2}\right\rangle+\left\langle z_{1}-z_{2}, x_{1}-x_{2}\right\rangle \\
& \leq M\left\|x_{1}-x_{2}\right\|^{2} .
\end{aligned}
$$


This implies that $F_{n}$ is semimonotone with constant $M$. Define $h_{n}(t, s)=$ $h(t, s)+2 n s$ for every $(t, s) \in[0, T] \times[0, \infty)$. We have

$$
\begin{aligned}
\left\|F_{n}(t, x)\right\| & =\sup _{y \in F(t, x)}\left\|A_{n} x+y\right\| \leq\left\|A_{n} x\right\|+\|F(t, x)\| \\
& \leq 2 n\|x\|+h(t,\|x\|)=h_{n}(t,\|x\|) .
\end{aligned}
$$

This shows that $F_{n}$ satisfies hypothesis (H2). Finally, suppose that $\left\{x_{m}\right\}_{1}^{\infty}$ is a sequence in $H$ which is convergent to $x$. If $y \in F_{n}(t, x)$, then there is a sequence $\left\{z_{m}\right\}_{1}^{\infty}$ in $H$ such that $z_{m} \in F\left(t, x_{m}\right), z_{m} \stackrel{w}{\rightarrow} z=y-A_{n} x \in F(t, y)$. Now, assuming $y_{m}=z_{m}+A_{n} x$, we have $y_{m} \stackrel{w}{\rightarrow} y$ in $H$ and $y_{m} \in F_{n}\left(t, x_{m}\right)$. Consequently, for every $n \in \mathbb{N}, F_{n}$ is hemicontinuous. By hypothesis (H2), $F$ takes the bounded sets in $[0, T] \times H$ to the bounded subsets of $H$. Since $A_{n}$ is a bounded operator, $F_{n}$ has this property also. Now, according to Theorem 3.5 (Theorem 3.5), there exists a unique generalized (classical) solution $x_{n}$ : $[0, T] \rightarrow H$ for the differential inclusion

$$
\left\{\begin{array}{l}
x^{\prime}(t) \in F_{n}(t, x(t)), \quad t \in[0, T] \\
x(0)=\xi
\end{array}\right.
$$

Hence, $x_{n}$ is a strong solution of the integral equation

$$
x(t)=\xi+\int_{0}^{t} A_{n} x(s) d s+\int_{0}^{t} v(s) d s,
$$

where $v \in S_{F, x}^{1}$. The solution $x_{n}$ is of the form

$$
x_{n}(t)=e^{A_{n} t} \xi+\int_{0}^{t} e^{A_{n}(t-s)} v(s) d s, \forall t \in[0, T] .
$$

Here $v_{n} \in S_{F, x_{n}}^{1}$ for all $n \in \mathbb{N}$. Since $D\left(A_{n}\right)=H, x_{n}$ is a classical solution and we have $\frac{d}{d t} x_{n}=A_{n} x_{n}+v_{n}, x_{n}(0)=\xi$. By the Hille-Yosida theorem, we know that $S(t) x=\lim _{n \rightarrow \infty} e^{A_{n} t} x$ for all $x \in H$ and moreover, the convergence is uniform on $[0, T]$. We conclude by Energy-Type inequality in [25] that, the sequence of approximate solutions $\left\{x_{n}\right\}_{n=1}^{\infty}$ is bounded by a constant which depends only on $M$ and $T$; in other words, we have

$$
\left\|x_{n}(t)\right\|^{2} \leq \gamma_{T, M}, \quad \forall t \in[0, T], \quad n \in \mathbb{N},
$$

where

$$
\gamma_{T, M}=e^{2 M+1}\left(\|\xi\|^{2}+\int_{0}^{T} h^{2}(t, 0) d t\right) .
$$

On the other hand, since $v_{n} \in S_{F, x_{n}}^{1}$, we have $\left\|v_{n}(t)\right\| \leq h\left(t, \gamma_{T, M}\right)$ for a.e. $t \in$ $[0, T]$. Therefore, we can assume that $\left\{v_{n}\right\}_{n=1}^{\infty}$ is weakly convergent to $v$ in $L^{1}(0, T ; H)$ and so, there exists a sequence $\left\{w_{n}\right\}_{n=1}^{\infty}$ of convex combinations of $\left\{v_{n}\right\}$ such that $w_{n} \rightarrow v$ in $L^{1}(0, T ; H)$ as $n \rightarrow \infty$. We extract a subsequence 
of it (denoted by the same symbol) which is a.e. convergent to $v$. Since, $F$ has close convex values, $w_{n} \in S_{F, x_{n}}^{1}$. Define

$$
y_{n}(t)=e^{A_{n} t} \xi+\int_{0}^{t} e^{A_{n}(t-s)} w_{n}(s) d s
$$

and

$$
x(t)=S(t) \xi+\int_{0}^{t} S(t-s) v(s) d s,
$$

for all $t \in[0, T]$. For every $z \in H$ we obtain

$$
\begin{aligned}
& \left\langle z, y_{n}(t)-x(t)\right\rangle \\
= & \left\langle z, e^{A_{n} t} \xi-S(t) \xi\right\rangle+\left\langle z, \int_{0}^{t} e^{A_{n}(t-s)} w_{n}(s)-S(t-s) v(s) d s\right\rangle \\
= & \left\langle z, e^{A_{n} t} \xi-S(t) \xi\right\rangle+\int_{0}^{t}\left\langle z, e^{A_{n}(t-s)} w_{n}(s)-S(t-s) v(s)\right\rangle d s .
\end{aligned}
$$

By the dominated convergence theorem, we conclude that $y_{n}(t) \stackrel{w}{\rightarrow} x(t)$ as $n \rightarrow$ $\infty$ for all $t \in[0, T]$ and since the sequence is integrably bounded, $y_{n} \stackrel{w}{\rightarrow} x$ in $L^{1}([0, T] ; H)$. Eventually, we use again Lemma 3.4 and conclude from the semimonotonicity of $G$ that $v \in G x$.

\section{Example}

In this section, we give an example to illustrate the application of the results obtained in this paper.

Assume that $\Omega$ is an open bounded subset of $\mathbb{R}^{2}$ with boundary $\partial \Omega$ of class $C^{2}$. Consider the second order differential inclusion

$$
\frac{\partial^{2} u}{\partial t^{2}} \in F\left(t, x, u, \frac{\partial u}{\partial t}\right),
$$

with the boundary condition $u(t, x)=0$, for each $(t, x) \in[0, T] \times \partial \Omega$ and initial conditions $\frac{\partial u}{\partial t}(0, x)=\psi(x), u(0, x)=\varphi(x)$. Here, $\varphi \in H_{0}^{1}(\Omega), \psi \in L^{2}(\Omega)$ and the Caratheodory multivalued map $F:[0, T] \times \Omega \times \mathbb{R}^{2} \rightarrow P_{b, c, v}(\mathbb{R})$ satisfies the following hypotheses:

(I) There is a constant $M>0$ such that for every $t \in[0, T], u_{1}, u_{2} \in \mathbb{R}$ and $v_{1}, v_{2} \in L^{2}(\Omega)$, we have

$$
\int_{\Omega}\left(\psi_{1}(x)-\psi_{2}(x)\right)\left(v_{1}(x)-v_{2}(x)\right) d x \leq M\left\|v_{1}-v_{2}\right\|_{L^{2}(\Omega)}^{2},
$$

in which the functions $\psi_{1}, \psi_{2}: \Omega \rightarrow \mathbb{R}$ are such that

$$
\psi_{1}(x) \in F\left(t, x, u_{1}, v_{1}(x)\right)
$$

and $\psi_{2}(x) \in F\left(t, x, u_{2}, v_{2}(x)\right)$ for all $x \in \Omega$. 
(II) There is an increasing continuous function $k(t)$ such that

$$
\|F(t, x, u, v)\|^{2} \leq k(t)\left(|u|^{2}+|v|^{2}\right)
$$

for all $(t, x, u, v) \in[0, T] \times \Omega \times \mathbb{R}^{2}$.

(III) For any sequence $\left\{u_{n}\right\}_{n=1}^{\infty}$ in $H_{0}^{1}(\Omega)$ which is convergent to $u$ and sequence $\left\{v_{n}\right\}_{n=1}^{\infty}$ in $L^{2}(\Omega)$ which is convergent to $v$, if there exists a function $z: \Omega \rightarrow \mathbb{R}$ such that $z(x) \in F(t, x, u(x), v(x))$ for all $t \in[0, T]$, then one can find a sequence of functions $z_{n}: \Omega \rightarrow \mathbb{R}$ such that $z_{n} \stackrel{w}{\rightarrow} z$ in $L^{2}(\Omega)$ and moreover, $z_{n}(x) \in F\left(t, x, u_{n}(x), v_{n}(x)\right)$.

Now, we consider the Hilbert space $H=H_{0}^{1}(\Omega) \times L^{2}(\Omega)$ with the inner product

$$
\left\langle\left(u_{1}, v_{1}\right),\left(u_{2}, v_{2}\right)\right\rangle_{H}=\int_{\Omega} u_{1}^{\prime}(x) u_{2}^{\prime}(x) d x+\int_{\Omega} u_{1}(x) u_{2}(x) d x+\int_{\Omega} v_{1}(x) v_{2}(x) d x .
$$

We define the multivalued map $G$ on $[0, T] \times H$ by $G(t, z)(x)=\{0\} \times F(t, x, z(x))$. Then, $G$ is a Caratheodory multivalued map with closed and convex values. It is easy to see that $G$ satisfies hypotheses $(H 1)-(H 3)$. Therefore, defining $z(t) x=\left(u(t, x), \frac{\partial u}{\partial t}(t, x)\right)$, we can reduce the above second order differential inclusion to the equivalent first order differential inclusion

$$
\left\{\begin{array}{l}
z^{\prime}(t) \in G(t, z(t)) \\
z(0)=(\varphi, \psi)
\end{array}\right.
$$

Hence, by Theorem 3.5 , it has a unique mild solution $u \in C\left([0, T] ; H_{0}^{1}(\Omega)\right)$ such that $\frac{\partial u}{\partial t} \in C\left([0, T] ; L^{2}(\Omega)\right)$ almost everywhere on $[0, T]$.

\section{References}

[1] R. P. Agarwal, M. Meehan, and D. O'Regan, Fixed Point Theory and Applications, Cambrige, 2001.

[2] J. P. Aubin and A. Cellina, Differential Inclusions, Springer-Verlag, 1984.

[3] J. P. Aubin and H. Frankowska, Set Valued Analysis, Birkhauser, 1990.

[4] R. Aumann, Integral of set-valued functions, J. Math. Anal. Appl. 12 (1965), 1-12.

[5] D. Barcenas and W. Urbina, Measurable multifunctions in nonseparable Banach spaces, SIAM J. Math. Anal. 28 (1997), no. 5, 1212-1226.

[6] A. Bressan, On the qualitative theory of lower semicontinuous differential inclusions, J. Differential Equations 77 (1989), no. 2, 379-391.

[7] _ Selections of Lipschitz multifunctions generating a continuous flow, Differential Integral Equations 4 (1991), no. 3, 483-490.

[8] A. Bressan and Z. Wang, Classical solutions to differential inclusions with totaly disconnected right-hand side, J. Differential Equations 246 (2009), no. 2, 629-640.

[9] F. E. Browder, Non-linear equations of evolution, Ann. of Math. 80 (1964), 485-523.

[10] _ Nonlinear initial value problems, Ann. of Math. 81 (1965), 51-87.

[11] A. Cellina, Multivalued differential equations and ordinary differential equations, SIAM J. Appl. Math. 18 (1970), 533-538.

[12] — A view on differential inclusions, Rend. Semin. Mat. Univ. Politec. Torino. 63 (2005), no. 3, 197-209.

[13] K. Deimling, Nonlinear Functional Analysis, Springer-Verlag, 1985.

[14] N. Dunford and J. T. Schwartz, Linear Operators. I. General Theorey, Interscience Publishers, Inc., New York, 1958. 
[15] A. F. Filippov, Classical solutions of differential equations with multi-valued right-hand side, SIAM J. Control 5 (1967), 609-621.

[16] H. Frankowska, Set-valued analysis and control theory (monograph), Birkhauser, (in prepatation).

[17] A. Granas and J. Dugundji, Fixed point Theory, Springer-Verlag, 2003.

[18] S. Hu and N. S. Papageorgiou, Handbook of Multivalued Analysis. Vol. I, Kluwer Academic, 1997.

[19] R. Jahanipur, Stability of stochastic delay evolution equations with monotone nonlinearity, Stoch. Anal. Appl. 21 (2003), no. 1, 161-181.

[20] _ Nonlinear functional differential equations of monotone-type in Hilbert spaces, Nonlinear Anal. 72 (2010), no. 3-4, 1393-1408.

[21] M. Kamenskii, V. Obukhovski, and P. Zecca, Condensing multivalued maps and semilinear differential inclusion in Banach spaces, De Gruyter Ser. Nonlinear Anal. Appl. 7, Walter de Gruyter, Berlin-New York, 2001.

[22] A. Lasota and Z. Opial, An application for the Kakutani-Ky Fan theorem in the theory of ordinary differential equations, Bull. Acad. Polon. Sci. Sér. Sci. Math. Astronom. Phys. 13 (1965), 781-786.

[23] A. Pazy, Semigroup of Linear Operators and Applications to Partial Differential Equations, Springer-Verlag, 1983.

[24] X. Xue and J. Yu, Periodic solutions for semi-linear evolution inclusions, J. Math. Anal. Appl. 331 (2007), no. 2, 1246-1262.

[25] B. Z. Zangeneh, An energy-type inequality, Math. Inequal. Appl. 1 (1998), no. 3, 453461.

Hossein Abedi

Department of Mathematical Sciences

UNIVERSITY OF KASHAN

87317-51167, Ravand Road, Kashan, Iran

E-mail address: h.abedi7@gmail.com

RuHollah Jahanipur

Department of Mathematical Sciences

UNIVERSITY OF KASHAN

87317-51167, RAVAND RoAd, Kashan, IRAN

E-mail address: jahanipu@kashanu.ac.ir 